\title{
Saberes que conectan con el poder durante el parto: la partería tradicional en Morelos (México)*
}

\section{Knowledge that Connects with Power During Childbirth: Traditional Midwifery in Morelos (Mexico)}

\author{
ESTER BOTTERI** \\ JACQUELINE ELIZABETH BOCHAR PIZARRO***
}

\begin{abstract}
This article reports an investigation carried out in Morelos on traditional Mexican midwifery, medicine composed of different tools that contribute to a harmonious pregnancy. A qualitative ethnographic research, especially accompanying midwife Angelina Martinez Miranda through the method of observation and participant perception and interviews with women who gave birth in this traditional way to different midwives in Mexico. Key words: cosmovision, traditional medicine, empowerment, women
\end{abstract}

\begin{abstract}
Resumen
Este artículo da cuenta de una investigación realizada en Morelos sobre la partería tradicional mexicana, medicina compuesta por diferentes herramientas que contribuyen a un embarazo y parto armoniosos. Es una investigación etnográfica cualitativa en la que de manera especial se acompañó a la partera Angelina Martínez Miranda, a través del método de la observación participante y con entrevistas a mujeres que parieron de esta forma tradicional con diferentes parteras en México.
\end{abstract}

Palabras clave: cosmovisión, medicina tradicional, empoderamiento, mujeres

\section{Introducción}

$\mathrm{E}$ 1 objetivo de este artículo $^{1}$ es mostrar la posibilidad de empoderamiento (Murguialday, Pérez de Armiño y Eizaguirre, 2001) de las mujeres a través de la medicina de la partería tradicional mexicana y las condiciones que propician un buen parto mediante las diferentes herramientas que la componen: las sobadas, el rebozo, la medicina placentaria y el cierre posparto, poniendo de manifiesto cómo tales herramientas favorecen lo que se suele llamar continuum en el cuidado (Liedloff, 2009).

* Artículo recibido el 14/11/17 y aceptado el 06/06/18.

** Universidad de Bolonia. Via Zamboni, 33, 40126 Bologna BO, Italia <esterbotteri91@gmail.com>. orcid: https://orcid. org/0000-0001-9255-1413.

*** Universidad Autónoma del Estado de Morelos, Centro de Investigación en Ciencias Sociales y Estudios Regionales. Av. Universidad núm. 1001, col. Chamilpa, 62209, Cuernavaca, Morelos, México <jacquiebopi@gmail.com>. oRciD: https:// orcid.org/0000-0002-6526-1901.

${ }^{1}$ Este artículo es resultado de la investigación etnográfica para la tesis de maestría en Antropología y Etnología de la Universidad de Bolonia de Ester Botteri, con la tutoría y codirección de las doctoras Jacqueline Bochar Pizarro (Universidad Autónoma del Estado de Morelos) y Zelda Franceschi (Universidad de Bolonia). La investigación de campo fue realizada en Tepoztlán, Morelos, México, de enero a abril de 2017. 
La investigación trata el proceso de embarazo y parto por parteras tradicionales recurriendo a la antropología activista, una de las "estrategias metodológicas $\mathrm{y}$ formas feministas alternativas de trabajo aplicado y de intervención social que se proponen crear puentes entre la academia y los actores sociales" (Suárez, Martín y Hernández, 2008: 13).

Se echó mano de una metodología interdisciplinaria entre la psicología, la antropología del nacimiento y teorías feministas, la cual alude a un análisis antropológico crítico que se apoya en la herramienta etnográfica cualitativa y se acompaña de la psicología social para poner el foco en los procesos subjetivos de las mujeres y sus emociones. Se abordó la afectación (Bochar Pizarro, 2017) que la práctica de la partería tradicional produce en el cuerpo y la mente de las mujeres, desde la contribución de las teorías feministas que evidencian a las normas como investimientos emocionales (Cavarero, 1995) que causan efectos en los cuerpos (Ahmed, 2017).

Es una investigación etnográfica exploratoria con parteras tradicionales, en particular con Angelina Martínez Miranda, a quien se acompañó y dio seguimiento. ${ }^{2}$ Se trabajó con el método de investigación acción participativa (Balcazar, 2003), y se realizaron cuatro entrevistas a mujeres seleccionadas al azar con la técnica de bola de nieve (Elorza Pérez-Tejada, 2008), que parieron con partera tradicional.

La partería tradicional a la cual hacemos referencia obedece a los saberes indígenas empíricos transmitidos de generación en generación, pero que también han adquirido mujeres mestizas a través de la práctica y el aprendizaje con las parteras indígenas. En Morelos, las parteras incorporan conocimientos de la biomedicina (Menéndez, 1991), sin dejar de lado sus saberes ancestrales, sobre todo los relativos a la herbolaria, la sobada, el uso del rebozo y el cierre posparto. Su participación en los cuidados de la salud maternoinfantil data de la época prehispánica (Jiménez, 2013).

Importa subrayar que en México se ha dado una coyuntura en la que el activismo ha promovido el derecho al parto humanizado; sin embargo aún no se ha logrado rescatar a la partería tradicional, y ni siquiera colocarla en un lugar significativo. ${ }^{3}$ Las parteras tradicionales están obligadas a certificarse (en diferentes campañas de capacitación), pero no se observa que sus técnicas se legitimen ni se autorice su práctica dentro de la medicina alopática (Menéndez, 1991). Se han realizado acciones para capacitar a futuras madres, y con el Proyecto Marco: Modelo Integral de Partería (2016-2018), diseñado por el Instituto Nacional de Salud Pública, se busca prevenir la muerte materna y mejorar la atención; no obstante, todavía no se reconoce el valor del saber tradicional de la partería.

\section{La perspectiva de la antropología médica y de la antropología del nacimiento}

Los primeros trabajos de antropología médica en México fueron los de Manuel Gamio (1922) sobre el mejoramiento de vida de los pobladores del valle de Teotihuacán. En la década de los cincuenta fueron relevantes los aportes del médico y antropólogo Gonzalo Aguirre Beltrán (1955), quien desarrolló la teoría del relativismo cultural antropológico, y más adelante se sumaron otras contribuciones de la biomedicina, las medicinas subalternas y la autoatención (Menéndez, 1980). Antes de esto, para comprender las medicinas de los otros, los antropólogos usaban la biomedicina como único método en las investigaciones etnográficas. Cuando la antropología comienza a tener presente su contexto social, la biomedicina se define como un sistema cultural específico (Quaranta, 2006).

Con el nacimiento de la antropología médica se empezaron a investigar de forma analítica las categorías interpretativas de la biomedicina y cómo éstas se relacionan con la experiencia del paciente. El primer experimento tuvo lugar en la Escuela de Harvard, fue impulsado por Arthur Kleinman, quien estudió la importancia de la experiencia de la enfermedad del paciente y su familia (illness) (Kleinman, 1988). La biomedicina explica la enfermedad en términos de alteración del funcionamiento de un órgano (desease), ignorando la experiencia de vida del afectado (Good y DelVecchio Good, 1982). Desde esta incapacidad del sistema médico hegemónico de detenerse en y atender las vivencias del paciente se desarrolló la llamada "no adherencia al tratamiento", que significa la no participación en el proceso de la curación. Dicho de otra forma, los médicos hablan de la enfermedad en un lenguaje sectorial, mientras las personas hablan desde lo que han vivido (Quaranta, 2006: 16). Como afirma el antropólogo Good (2006), el modelo biomédico

2 Partera tradicional que vive en la ciudad de Tepoztlán, Morelos. Ella estimula a las mujeres a seguir sus propias decisiones en relación con sus cuerpos y la forma de parir.

3 Secretaría de Salud, "Esquema metodológico para incorporar la perspectiva de género mediante el enfoque intercultural" <https: / www.gob.mx/cms/uploads/attachment/file/29315/EsquemaGeneroIntercultural.pdf> [15 de mayo de 2018]. 
reduccionista piensa las enfermedades como de origen biológico-orgánico, sin tener en cuenta la interacción entre lo biológico, lo cultural y lo social y, menos aún, la intervención de las emociones y la relación médicopaciente en el proceso de la cura.

En particular, el reto de la antropología médica fue superar el dualismo cartesiano típico de la biomedicina y concebir el cuerpo como mindful body (ScheperHughes y Lock, 1987), el cuerpo consciente, que activamente se relaciona con el mundo y deja de ser una entidad pasiva y dividida entre el pensamiento y el cuerpo. Así, la enfermedad, en cuanto manifestación del desorden personal, se convierte en una modalidad de resistencia al orden cultural y a las relaciones de poder (Scheper-Hughes y Lock, 1987). El cuerpo femenino es un lugar de incorporación de fuerzas económico-político-sociales (Butler, 2002), pero también es el principal instrumento metodológico, epistemológico y político de expresión de la subjetividad (Esteban, 2011 ). Según la perspectiva de la antropología médica, el cuerpo permanece pasivo ante las fuerzas externas que lo enferman; sin embargo, es capaz de resistirse a las fuerzas sociales y culturales que lo oprimen y tener el control y la agencia (Esteban, 2004; Juliano, 2017), amén de tomar decisiones sobre sí mismo.

En esta investigación se observa que los recursos propios (biológicos y psicológicos) resultan de gran beneficio para las mujeres embarazadas y parturientas. Además, la psicología ha sido una disciplina que ha investigado en esos recursos personales, sobre todo basada en que la enfermedad es un proceso en el cual intervienen activamente las emociones, y que el camino hacia la salud dependerá de que las emociones involucradas se encaucen y se procesen (Dethlefsen y Dahlke, 2005). En el caso que nos ocupa, se constató que la confianza en parir de manera natural (que es un saber transmitido de una generación a otra) se favorece cuando las mujeres han transitado por un proceso de sanación del vínculo con sus propias madres y aceptan lo que su madre pudo hacer y dar, sin juzgar sus comportamientos (Rodrigáñez Bustos, 1995), y procesan el duelo por la hija que ahora se transforma en madre y se descoloca del lugar de la que demanda, para ser ahora quien brinda al nuevo ser el cuidado, la protección y el amor necesarios para la sobrevivencia.

\section{Crítica a la biomedicina y a los saberes patriarcales}

En el caso del cuerpo de las mujeres, la incapacidad de curación se acentúa a través de la cultura patriarcal, que siempre ha desvalorizado y minimizado el saber de las mujeres (Moore, 1994; Butler, 2002). En particular durante el embarazo, la biomedicina coloca la autoridad en las manos de los médicos (Oakley, 1985; Davis-Floyd y Sargent, 1996) y no ha reflexionado a suficiencia sobre la importancia que puede tener un parto natural para la subjetividad de una mujer madre cuando se le otorga la posibilidad de manejar sus propios recursos biológicos (Rodrigáñez Bustos, 2007). La mujer que pare en casa reafirma la confianza en sí misma y en su cuerpo, lo cual tendrá repercusiones en la relación madre-hijo (Odent, 1984; Gaskin, 2002). Por el contrario, cuando se le quita la posibilidad de valorar su experiencia de parto se convierte en un instrumento en las manos de la tecnología, entregada a confiar en un modelo hospitalario (Bestetti y Regalia, 2007; Regalia y Bestetti, 2010).

Si miramos atrás, en muchos contextos culturales, el parto ha sido considerado un momento de gran trascendencia, donde la mujer está conectada con la fuerza de las antepasadas (Gimbutas, 1989; Luciani, 2008). El nacimiento se convierte en el primer y mayor acto de espontaneidad, donde se despliega toda la fuerza para venir a este mundo (Moreno, 1977). Según médicos como Olza y Lebrero (2005), la tipología de parto vivido marcará al niño en su desarrollo psíquico, impulsándolo a la vida con confianza o con temor.

Es interesante en este sentido el análisis genealógico del filósofo Michel Foucault (1988: 54), que evidencia cómo el hospital, centro del poder de la biomedicina, se ha vuelto un lugar donde se reúnen los enfermos; así, el médico se tornó en la persona que "restablecía el orden dentro de un desorden dictado por la naturaleza", lo que el estudioso llamó la medicalización del hospital, que produjo el efecto de quitar el poder al enfermo sobre su propio cuerpo y dar al sistema biomédico la autoridad sobre los cuerpos.

Lo mismo pasó con las mujeres embarazadas y parturientas. A partir de una perspectiva de la antropología del nacimiento, la antropóloga Emily Martin estudió las raíces culturales del control patriarcal biomédico que concibe el cuerpo de la mujer como "el espacio de intervención de la tecnología” (1987: 31-32).

Según Casilda Rodrigáñez Bustos, para la cultura patriarcal, "la mujer es una histérica y el bebé no siente y no entiende" (1995: 60); por lo tanto, impedir un parto fisiológico evita el desarrollo de la libido materna, que es incompatible con la sociedad patriarcal. La renuncia a tener un parto con placer se suma a la cadena de renuncias al placer que exige el sistema. "Parirás con dolor" es el mandato que se hace ley y se introyecta como parte de la imposición genérica que niega a las mujeres sentir placer, porque es necesario domarlo 
para hacerla dócil y sumisa. La consecuencia de esto es lo que la psicoanalista llama útero espástico: el útero se convierte en un envase, incapaz de responder a los estímulos externos (Rodrigáñez Bustos, 2007: 15). No obstante, fisiológicamente el útero funciona como una caja de resonancia, que transmite emociones, sentimientos e información a todo el cuerpo y no tiene una mera función reproductiva.

En conclusión, podemos decir que el cuerpo de la mujer fue una invención de un modelo patriarcal que fue cambiando según los ideales de cada época, y la biomedicina representa el modelo oficial de este ejercicio (Laqueur, 1994).

La partería tradicional es un modelo que garantiza una atención integral sustentada en la escucha del cuerpo, que refuerza el empoderamiento de las mujeres y da valor a su experiencia personal a través de la pregunta disparadora: “¿Tú, qué quieres, mamita?". ${ }^{4}$ Esta modalidad de trabajo de fortalecimiento físico y emocional, mediante una escucha profunda de la mujer que revalora la decisión de la madre, marca la diferencia respecto de la atención de tipo médico asistencial, donde la decisión del parto queda sólo en manos de los médicos y el personal sanitario (DavisFloyd, 1992).

La pregunta es esencial desde el punto de vista feminista, ya que uno de los cuestionamientos más agudos a la medicina androcéntrica es la expropiación de la salud (Gervás y Pérez, 2015) y, en especial, del saber de las mujeres (Laqueur, 1994).

En consecuencia, la figura de la partera tradicional emerge como una clave con una profunda sabiduría (Luciani, 2008).

Históricamente, la partería había sido un saber en las manos de las mujeres, transmitido por tradición oral, hasta que se convirtió en un problema para la Iglesia católica (Dávalos, 1996), la cual comenzó a preocuparse por las ánimas de los fetos que morían durante el parto, y por el control de los nacidos vivos. Fue así que las parteras empezaron a ser asistidas por los párrocos, quienes bautizaban a los recién nacidos. Hacia 1768 se creó la primera escuela de obstetricia dirigida por médicos y cirujanos y se eliminaron las prácticas de partería por juzgarse peligrosas (León, 1910). Los médicos pensaban que las modalidades tradicionales de parto (en cuclillas o sentadas) eran “costumbres para mujeres ignorantes" (León, 1910:
45) y las sustituyeron por la posición litotómica. ${ }^{5}$ Así se instauró un saber sistemático y académico que abrió las puertas a la biomedicina (Foucault, 1988).

Por otro lado, el "parto en casa" está ligado al proceso de continuum en el nacimiento (Liedloff, 2009), ya que se pare en un lugar de confianza y que, además, será el hogar donde el bebé se va a desarrollar. Continuum significa proceso de continuidad, fluido, sin cortes físicos ni emocionales. Según el estudio de Guattrocchi (2011), las parteras de la comunidad de Kaua (Yucatán) son promotoras de una salud "que no corta", ${ }^{6}$ a diferencia de la perspectiva médica y hospitalaria donde se practica la cesárea y la episiotomía.

A pesar de la persecución, la partería tradicional en México ha resistido y sobrevivido como saber heredadoy transmitido oralmente (Carrillo, 1999; Freyermuth y Montes, 2000).

Importa resaltar que, en México, las parteras han recibido y continúan recibiendo cursos de capacitación sobre temas de riesgo durante el embarazo y están conectadas a servicios de ambulancias estatales que recurren de inmediato en caso de complicaciones. En Morelos, el servicio se llama Centro Regulador de Urgencias Médicas (CRUM). Empero, y a pesar de los esfuerzos realizados para disminuir la muerte materna (Luna, Muños y Freyermuth, 2017), mucho resta por hacer para mejorar la calidad y las condiciones del servicio para un parto natural y placentero.

En incontables ocasiones las comunidades están alejadas de los hospitales y los centros de salud, por lo cual la partera se convierte en la única opción. Dadas sus características infraestructurales y arquitectónicas, el hospital no es el lugar idóneo para parir y, además, no en todos los pueblos existen casas de salud para el parto y los hospitales están saturados. En algunas ciudades se están construyendo casas de parto, pero con ínfimos recursos. ${ }^{7} \mathrm{Ha}$ aumentado la atención de los partos en los hospitales, mas esto no necesariamente favorece la vivencia de un "buen parto". Durante una observación participante en el hospital del Instituto Mexicano del Seguro Social en Cuernavaca, durante 2017, se pudo constatar que en una sala de preparto estaban ingresadas 11 mujeres, con asistencia de un ginecólogo por turno; nueve de ellas en preparto y dos para legrados vaginales. Dicha sala no permite el acceso a acompañantes, por razones de higiene que se deben preservar. También está

4 Pregunta que utiliza la partera Angelina Martínez Miranda.

5 Posición ginecológica que se usa para parir en el hospital.

Que no practica cesáreas.

7 Información recabada en entrevistas con directores de diferentes programas de salud; uno referido a salud intercultural y otro a derechos reproductivos en el estado de Morelos. 
prohibido caminar en la sala (por falta de espacio), y no se autoriza otra postura que no sea la horizontal. ${ }^{8}$ Lo anterior da cuenta de que la disminución de la muerte materna no repercutió en la calidad del servicio, el cual podría mejorarse si se implantara la atención específica de los partos en casas de maternidad debidamente equipadas, acondicionadas y atendidas por parteras.

\section{Medicina y cosmovisión de las parteras tradicionales}

La medicina de las parteras alude a una cosmovisión del cuerpo como caliente-frío, cerrado-abierto, donde el cuerpo se concibe como un microcosmos relacionado con un macrocosmos (universo) y con un centro: el ombligo-tipté (López Austin, 1980). En este punto del cuerpo (tipté) comienzan todas las venas y las arterias, pero no es fijo, pues recibe esfuerzos o fuerzas que lo desplazan, y necesita ser recompuesto a través de una manteada o una sobada (masaje manual).

En este sentido, es esencial la doctrina humorística de la cosmovisión (López Austin, 1980), donde se identifica una relación entre "calor" (asociado con masculinidad, envidia, rabia, agresividad) y "frío" (asociado con frialdad emotiva y feminidad). De hecho, naturalmente se considera a las mujeres de humor frío y, cuando pierde calor (por ejemplo después de una menstruación -porque con la sangre menstrual se pierde calor-o durante el alumbramiento), su cuerpo resultará más frío (López Austin, 1980; Villa Rojas, 1985; Fagetti, 1998). Según la antropóloga Quagliariello (2014), esta dualidad no significa una oposición real (como es concebida en el contexto occidental), sino un balance térmico en equilibrio y en continuo cambio entre frío y calor, donde existen opciones para reequilibrar y que, en Mesoamérica, son tratados con sobada, rebozo, masaje, uso de plantas y de ingestiones calientes o frías.

En el ejercicio etnográfico de esta investigación se observó que durante el preparto y el parto se emplean plantas medicinales calientes y estimulantes: canela, jengibre, ruda ( $R$. graveolens), zoapatle (Montanoa tomentosa Cerv.) y epazote (Dysphania ambrosioides), entre otras. Como dice Angelina: "La regla y el parto impli- can la pérdida de calor del cuerpo, por lo cual se necesita devolverlo a través del uso de estas plantas calientes".

Durante la labor de parto se da un té de ruda y chocolate para restituir el calor y producir la dilatación, o se realizan baños de temazcal, ${ }^{9}$ sitio ideal para dilatar el útero.

Después del parto también se usan plantas medicinales como el zoapatle, que ayuda a que salga la placenta o a detener la hemorragia. Para desgarros y prevenir infecciones se indica árnica (Arnica montana). En el posparto, con objeto de aumentar la leche, se da anís estrellado y agua de arroz. La placenta juega un papel fundamental para estimular la producción de la leche materna y para evitar la hemorragia. Por ello, algunas parteras tradicionales manifiestan que la ingestión de un trozo de placenta después del parto impide la hemorragia y favorece la producción de leche, además de que previene la depresión.

Otro elemento muy utilizado por la partería tradicional de Morelos es el rebozo, ${ }^{10}$ uno de sus usos es acomodar al bebé que viene de nalgas o transverso (sentado). Cuando esto sucede se recurre además a la sobada abdominal y al masaje en los pies, que mejoran la circulación.

Existe una diferencia entre el uso del rebozo para el masaje y la sobada; simbólicamente, el rebozo es "la extensión de las manos de la mamá que recoge y protege al bebé y también es las manos de la abuela que cuida después del parto y entrega toda la protección de su linaje”.

El rebozo se utiliza, asimismo, para realizar el cierre posparto ocho días después del alumbramiento, un ritual de unificación y sanación. Consiste en dar un baño de hierbas calientes de limpia: ruda, lavanda (Lavandula), romero (Rosmarinus officinalis), salvia (Salvia officinalis), santa maría (Tanacetum balsamita), caléndula (Calendula officinalis), y se hace una sobada para "reposicionar los órganos", en particular el útero, que sufrió una gran tensión y se mudó de lugar; luego se usa el rebozo para cerrar siete veces el cuerpo en diferentes lugares (cabeza, hombros, pechos, ombligo, cadera, rodillas, pies), esto se consigue con dos personas que al mismo tiempo empujan de los extremos apretando el cuerpo (manteada).

Este cierre tiene un fuerte significado simbólico que nos habla de la necesidad del cierre de los ciclos

8 Dicha observación fue parte de la investigación "Mujeres, saberes tradicionales y políticas públicas" financiada por el Programa para el Desarrollo Profesional Docente 2016-2018 y dirigida por la doctora Jacqueline Bochar Pizarro del Centro de Investigación en Ciencias Sociales y Estudios Regionales de la Universidad Autónoma del Estado de Morelos.

9 El temazcal es una construcción en forma de matriz, hecha de barro o carrizos. En él se ponen piedras calientes que producen vapor al contacto con el agua. Una vez que las personas están dentro para darse un baño de vapor se cierra la puerta. La gran utilidad de esta práctica después del parto es calentar los órganos que se enfriaron al parir.

${ }^{10}$ Faja típica de México. Mediante una técnica conocida con el nombre de manteada, la partera realiza una serie de movimientos con el fin de relajar a la mujer para que se sienta más cómoda y confiada. 
después de un evento importante, cualquiera que sea, en este caso el proceso de parto.

Según la experiencia de las mujeres entrevistadas, ${ }^{11}$ al término del cierre el cuerpo queda cerrado, fuerte, compuesto y centrado.

\section{Las experiencias de parto con partera: la vivencia de las mujeres}

No hay pruebas científicas que confirmen que una mayor medicalización mejora el parto, como bien señala el ginecólogo Michel Odent "el parto es un procedimiento involuntario que involucra las estructuras cerebrales primarias, esto es lo contrario a la idea de que la mujer parturienta tiene que 'aprender a parir' " (1992: 13). La partera acompaña a la mujer a reconocer su saber en el ciclo sexual y reproductivo (antes, durante y después del nacimiento), de aquí la idea de continuum del proceso, y la decisión de la mujer sobre su propio cuerpo juega un papel determinante en el buen desarrollo del proceso.

Merece la pena resaltar que durante la investigación se encontró que quienes parieron con las parteras evidencian el continuum del cuidado (Liedloff, 2009), es decir, la no interrupción y la división de la cura entre las diferentes fases del parto (preparto/parto/ posparto). De acuerdo con testimonios de algunas entrevistadas, el parto natural con partera tradicional produjo la toma de conciencia de esta continuidad. Según F., una mamá de 30 años:

Mi partera me siguió mucho durante el embarazo y me cuidó muy bien, cuando fue tiempo de dar a luz me acompañó con amor y seguridad, haciéndome masajes y dándome chocolate con ruda; esto permitió calentar mi útero, alcanzar el parto y también tranquilizarme. ${ }^{12} \mathrm{Mi}$ parto fue tan fuerte y maravilloso y yo sentía como un placer, así que amamantar enseguida a mi hijo fue algo natural y muy fuerte; también la partera esperó a la placenta y dejó que fuera mi esposo que la cortara, para él fue increíble y esto le permitió estar más cerca de mi hijo y de mí. Yo creo que vivir algo tan mágico te permite ser una mamá feliz y segura, que confía en la fuerza de la vida y del amor [entrevista con F., 1 de abril de 2017 , Tepoztlán].

De alguna forma, la partera tradicional oficia como acompañante terapéutico ${ }^{13}$ durante el embarazo y el parto; esto es, no sólo tiene conocimiento empírico sobre el cuerpo fisiológico sino también da seguimiento al proceso de cambio psicológico. Durante el alumbramiento se experimenta una conmoción que, según los testimonios de las entrevistadas, las parteras saben escuchar, canalizar, contener y valorar.

De hecho, la mamá muda de una condición de energía caliente (preparto y parto) a una de energía fría (posparto), durante la cual se vuelve muy vulnerable a los "malos aires" que entran desde el útero, por eso le es necesario cerrar con la práctica de la faja (López Austin, 1980), comúnmente llamada cierre posparto. Dice Angelina: "Es importante cerrar el útero a partir de la salida del bebé, esto permite a la madre estar protegida de los malos aires que se pueden manifestar en el cuerpo como frialdad y depresión posparto".

Otro continuum que se garantiza a través del acompañamiento con la partera es el del apego al bebé después del nacimiento. Como señala la antropóloga Jean Liedloff: "un bebé continuum desde el momento en que nace está siempre en contacto con el cuerpo de alguien. Antes de que el cordón umbilical se desprenda, la vida del bebé ya está llena de acción" (2009: 84). Para el recién nacido es necesario el contacto y el apego durante los primeros seis meses de vida (Leboyer, 1974; Weber, 2013).

El bebé se queda en el pecho de su madre durante largo tiempo después del parto, permitiendo que la prolactina, la hormona del hogar y de la nutrición, se desarrolle: "cuando una mujer amamanta todo el efecto de las hormonas del amor se dirigen hacia el hijo, que se convierte en el principal objeto de amor" (Odent, 1992: 95). Si esto no sucede es más difícil hacer este pasaje, la relación se complica y la producción de leche se reduce. En ese momento se generan las primeras experiencias de satisfacción, lo que dará origen al deseo (Freud, 1970 y 1985). El deseo se

${ }^{11}$ Véase la tesis de maestría de Ester Botteri "La medicina del cierre en el posparto: experiencia de mujeres mesoamericanas", Universidad de Bolonia, julio de 2017 <https: / www.unibo.it/it/servizi-e-opportunita/servizi-online/servizi-onlineper-studenti-1 / guida-servizi-online-studenti/ams-tesi-online>.

12 En la percepción de las parteras tradicionales, el uso de plantas calientes como la ruda o la salvia puede acelerar el parto porque da calor a los órganos, esto permite una mayor afluencia de sangre (López Austin, 1980).

${ }^{13}$ Este concepto se originó en el ámbito de la psicología durante los años sesenta en América del Sur, específicamente en Argentina y Brasil, cuando se reveló el movimiento antipsiquiátrico. El acompañante terapéutico es una figura que da continuidad a los cuidados del paciente en el ámbito de la salud mental. Si bien el concepto surgió para establecer nuevas formas de atención al paciente psiquiátrico, podría utilizarse para pensar la función que ejerce la partera con las embarazadas y parturientas, un cuidado que acompaña la salud física y mental. Para profundizar se recomienda ver Pichón Rivière y Ana Pampliega (2002). 
genera en ese contacto entre la madre y el bebé, en el espacio entre los cuerpos, el contacto, las caricias. El aroma, el canto, el abrazo producen las primeras representaciones psíquicas que, de acuerdo con la teoría psicoanalítica, son el inicio del psiquismo humano (Freud, 1977).

$\mathrm{El}$ apego inicial hacia la madre es el fundamento de la confianza en sí mismo, tal como lo ha investigado y postulado el psicoanálisis. Un bebé sin ese vínculo de apego incluso puede llegar a morir (Bowlby, 1958; Spitz, 1965).

Una madre de 30 años, que tuvo un parto por cesárea en hospital y uno posterior con partera, narra la frustración y el dolor que experimentó después del nacimiento de su primer hijo mediante cesárea y, con su relato, describe claramente la situación:

Después de una cesárea no esperada no vi a mi hijo por un tiempo largo, tres días, me lo quitaron diciendo que tenían que hacer exámenes porque él sufrió al nacer. $\mathrm{Ni}$ mi esposo ni nadie pudo verlo hasta tres días después, por mucho tiempo tuve culpa y sentí desprecio por mí misma y ese desprecio se proyectaba sobre el bebé, por eso no quise amamantarlo. Tengo una herida profunda que sané con mi segundo parto donde decidí buscar a una partera, parir en casa y amamantar a mi niño. Cuando nació mi segundo hijo fue mágico y lleno de amor en mi casa en un día de enero. Mi hijo no se separó de mí y
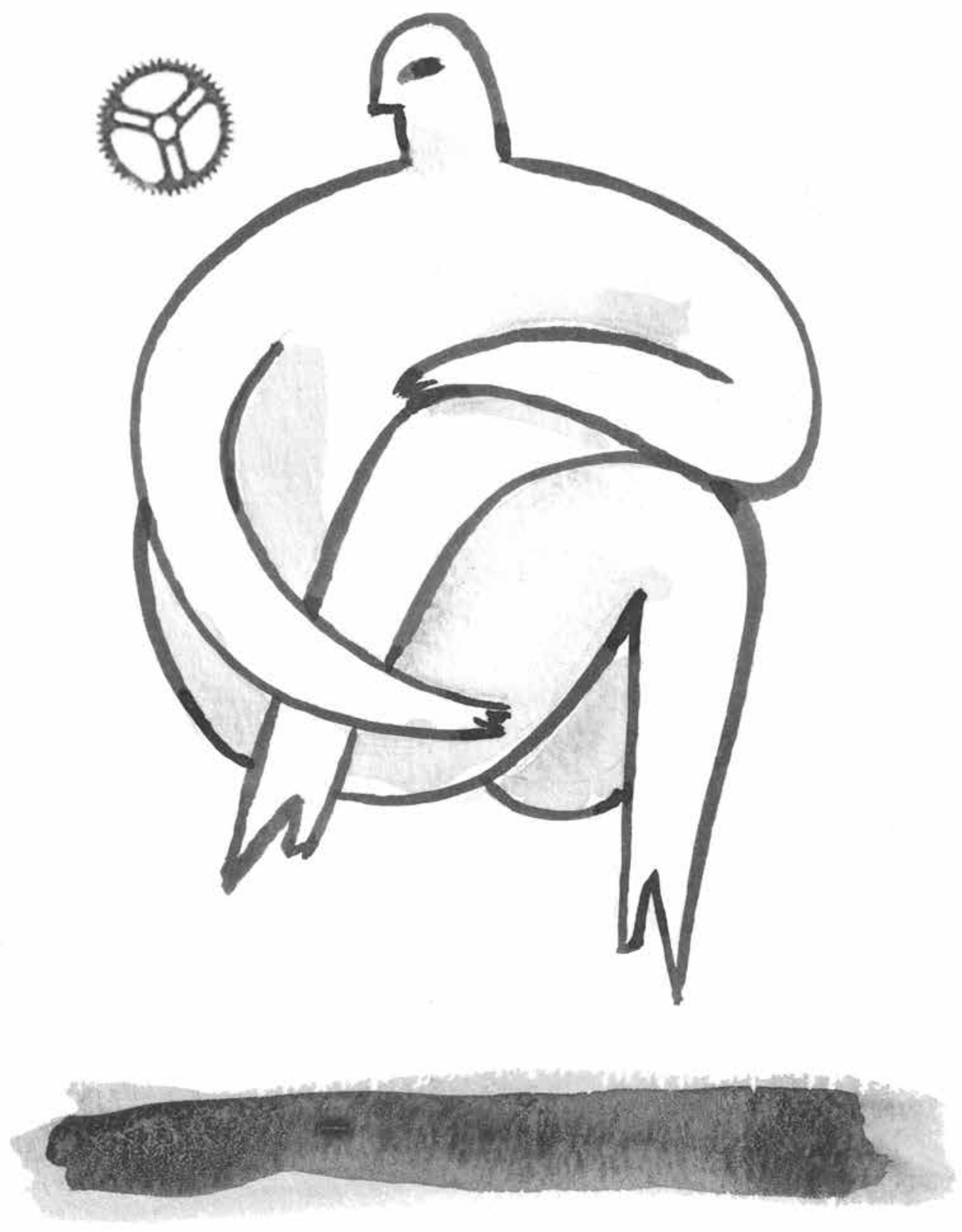
fuimos un único cuerpo por semanas. Esto me permitió expurgar culpas y tantos dolores respecto de mi primer parto [conversación y entrevista con M., 15 de abril de 2017, Tepoztlán].

Tal como afirma el médico Braibanti (1993), el ser humano requiere una exogestación (extracuidado), una gestación afuera de la matriz, que se realiza después del nacimiento, con un apego constante al pecho de la madre (igual que los marsupiales). Esto se debe a que los mamíferos necesitan una gestación que dura de 18 a 24 meses, mientras que el ser humano tiene una gestación de sólo nueve, por esta razón los meses que faltan no se consideran meses en los cuales el recién nacido sea independiente, sino que es un periodo en el cual la presencia de la madre y el apego son fundamentales. El bebé humano no sobrevive sin esos extracuidados indispensables.

G., de 38 años, nos narra un parto con apego negado en el hospital y uno segundo en casa:

Tuve un parto natural en el hospital, tuve complicaciones y hemorragia, me sacaron la niña y no la vi por dos días. Cuando regresé a mi casa con la niña no la reconocí, me dio mucha tristeza y culpa porque una parte de mí la rechazaba. Ninguna persona me ayudó con el posparto, ni me sostuvieron mucho mis hermanas, me sentí plena de culpa y sola, pero una parte de mí pensó: esto no me pasará otra vez. Cuando supe que estaba nuevamente embarazada decidí que tenía que ser en casa, en mi casa y con partera. Así fue que nació L., un niño de $4.230 \mathrm{~kg}$, todo fue muy fácil, la partera me apoyó mucho, me dijo que yo sola podía hacer todo. Ella sólo me sobó, me masajeó con el rebozo y me llevó a caminar. Ayudó también mi esposo a masajearme, a estar cerca de mis necesidades, y a confiar en la necesidad de su presencia en el parto. Cuando empezaron las contracciones mi partera me miró a los ojos y sólo dijo: "iPonte como quieras, haz lo que quieras!” —“¿Y cómo lo hago?” —“Tú sabes hacerlo y cómo hacerlo, tú puedes". Así que me puse como un tigre en cuatro patas y mi esposo me sostuvo mientras mi hija mayor me masajeaba los pies y cantaba conmigo, y entonces todo empezó a fluir y me quedé en casa 40 días escuchando mis necesidades y mi bebé. Me sentí empoderada y segura de ser una mamá en plena forma. Mi segundo parto sanó profundas heridas de mi niñez y de mi primer parto [conversación y entrevista con G., 10 de abril de 2017].
Un tercer elemento que las mujeres exteriorizan es su paso del lugar de la víctima culpable y silenciada (vivido durante la cesárea) a la mujer fuerte y atenta a sus necesidades durante el parto natural con partera.

F., una mamá de 40 años, dijo:

Mi cesárea fue traumática, no la esperaba, fue así que de pronto me llevaron a la sala operatoria, estaba sola: cuando salí me pareció que había pasado mucho tiempo, todo me dolía y me sacaron mi bebé dormida. El posparto fue difícil, estuve muy triste y muy melancólica. Mi esposo y mi mamá fueron conmigo, pero no me perdonaba no haberlo logrado. Para mi segundo parto contacté a una partera y todo fue muy diferente, yo estaba en su casa de parto con mis amigas, mi pareja, mi mamá y las dos parteras. Me sobaron, me masajearon y viví un trabajo de parto sin miedo y muy fluido: fue increíble. Sólo durante el alumbramiento en el coronamiento se pararon las contracciones y yo tuve mucho temor de que se repitiera lo que había vivido con mi primer parto. Intervino mi partera y me dijo: "llegaste hasta aquí, eres muy fuerte, ahora tienes que recoger todas las energías de tus ancestras y permitir a esta bebé salir: si tú quieres, tú puedes; eres una guerrera. Yo confío en ti, todos la estamos esperando".

La indicación de la partera estimuló a la parturienta, la habilitó en su saber.

Este hecho me permitió también adueñarme de mi posparto donde, con la ayuda de la partera que me sobó, me masajeó y cerró mi ciclo de parto, me sentí muy fuerte, invencible, lista para vivir en plenitud y conectada con todas las mujeres que parieron antes que yo. Nosotras, las mujeres, estamos plenas de poder [conversación con F., 16 de marzo de 2017, Cuernavaca].

La conexión con el saber ancestral y la profundidad de este conocimiento ${ }^{14}$ es un tema permanentemente invocado por las parteras tradicionales, porque se enfoca en los rituales tradicionales de la comunidad (Gonzales, 2012).

Ancestras son, en este caso, las mujeres que parieron, por linaje materno, antes que ellas, que se presentan como un ejemplo de fuerza y que permiten sacar el poder que cada mujer tiene. Interpretamos dicha conexión como un reconocimiento del saber que

${ }^{14}$ En Ecuador, por ejemplo, se subraya la importancia de las parteras y su sabiduría ancestral en el fomento de la salud de las mujeres embarazadas y de las niñas y los niños. Esto se establece en el "Manual de Articulación de prácticas y saberes de parteras ancestrales en el Sistema Nacional de Salud" (febrero 2017), norma del Ministerio de Salud Pública del Ecuador. 
está alojado en la memoria corporal (Esteban, 2011) de cada mujer y que hace posible que se empodere, que tome el poder. Cada mujer nace sabiendo parir sin importar si desea o no ser madre (Rodrigáñez Bustos, 2007). No obstante, este saber puede verse bloqueado por la aculturación occidental y la socialización en un sistema que promueve la vulnerabilidad, el sentimiento de indefensión y la expropiación del cuerpo.

\section{Conclusiones}

Durante las entrevistas realizadas las mujeres contaron sus historias enfocadas en el proceso de continuum empoderante que vivieron, y pusieron en evidencia que no hubo corte ni separación entre la fase del parto y el posparto; inclusive, en algunos casos, el primer parto hospitalario fue vivido como una experiencia de vulnerabilidad, donde sufrieron cesáreas o faltas de apego, lo cual trajo aparejado un sentimiento de culpa en relación con su rol materno, muy al contrario de lo experimentado en el proceso con parteras. Esto lo asociaron en concreto con la capacidad de la partera de empoderarlas, es decir, de devolverles el poder sobre sí mismas durante el proceso de embarazo-parto. La partera fue identificada como un canal que confía en las mujeres y en su capacidad de toma de las mejores decisiones.

La pregunta disparadora “¿Tú qué quieres?” (la cual se formula desde el preparto, cuando se consulta a la partera por primera vez) y la calidez del trato que reciben son los hechos que favorecen la confianza y la apropiación del proceso que propicia un parto natural gozoso.

La posibilidad que les otorga esta clase de parto tradicional refuerza el poder activo que las mujeres traen, el poder de parir de forma natural y espontánea. En el hospital los médicos mantienen distancia emocional de los cuerpos, vistos como objeto de trabajo y desprovistos de emociones, a la vez que son codificados en un territorio que puede ser invadido por la revisión sin consideración (Bestetti y Regalia, 2007).

Las experiencias nos hablan también de vivencias traumáticas previas, que fueron superadas mediante un parto natural acompañado de una partera y en la casa de la familia o en el lugar dispuesto por la madre. La mujer cuando es consultada y decide activamente cómo, con quién y dónde parir recupera su lugar de sujeto activo, se adueña de su cuerpo y se libera de los sentimientos de culpa y las emociones de rechazo hacia sí misma.

El parto con partera, tal como constatamos gracias a las entrevistas y la observación participante en el acompañamiento a la partera, permite retomar la autoconfianza y, además, contribuye a que la mujer sea capaz de sanar un trauma vivido anteriormente por una cesárea y recupere el placer, desmitificando el mandato divino de "Parirás con dolor".

\section{Fuentes}

Aguirre Beltrán, Gonzalo

1955 Programas de salud en la situación intercultural, Instituto Indigenista Interamericano, México.

Ahmed, SARA

2017 La política cultural de las emociones, Universidad Nacional Autónoma de México-Centro de Investigaciones y Estudios de Género, México.

BALCAZAR, FABRICIO E.

2003 "Investigación acción participativa (IAP): Aspectos conceptuales y dificultades de implementación", en Fundamentos en Humanidades, vol. IV, núms. 7-8, pp. 59-77<http:// www.redalyc.org/pdf/184/18400804.pdf> [ 15 de mayo de 2018].

Bestetti, Giovanna

y Anita Regalia (EDs.) 2007 Il dolore è... nel parto!, Mimesis, Milán.

Bochar Pizarro, Jacgueline Elizabeth

2017 "Intervención sociodramática y construcción de autonomía con mujeres afectadas por violencia", en Daniela Cerva Cerna (coord.), Varias miradas, distintos enfoques. Los estudios de género a debate, Universidad Autónoma del Estado de Morelos (Praxis Digital 14), México, pp. 173-195.

BOWLBY, JOHN

1958 "The Nature of the Child's Tie to His Mother", en International Journal of Psycho-Analysis, vol. 39, núm. 5, pp. 350-373.

Braibanti, LORENZO

1993 Parto e nascita senza violenza, Red Edizioni, Milán.

Butler, Judith

2002 Cuerpos que importan. Sobre los límites materiales y discursivos del "sexo", Paidós, Buenos Aires.

Carrillo, Ana María

1999 "Nacimiento y muerte de una profesión. Las parteras tituladas en México", en Dynamis. Acta Hispanica ad Medicinae Scientiarumque Historiam Illustrandam, núm. 19, pp. 167-190.

Cavarero, Adriana

1995 Ermeneutica della differenza sessuale, Universidad de Valencia (Eutopías: Documentos de Trabajo, 96), Valencia.

Dávalos, Marcela

1996 "El ocaso de las parteras", en Cuicuilco, vol. 2, núm. 6, pp. 195-211.

DAVIS-Floyd, RoBBIE

1992 Birth as an American Rite of Passage, University of California Press, Berkeley.

Davis-Floyd, Robbie E. y Caroline Sargent

1996 "The Social Production of Authorative Knowledge in Pregnancy and Childbirth", en Medical Anthropology Quarterly, vol. 10, núm. 2, pp. 111-120. 
Dethlefsen, THORWALD

Y RÜDIGER DAHLKE

2005 Laenfermedadcomocamino, Debolsillo, México.

Elorza Pérez-Tejada, Haroldo

2008 Estadística para las ciencias sociales, del comportamiento y de la salud, Cengage Learning, México.

Esteban, Mari Luz

2004 Antropología del cuerpo. Género, itinerarios corporales, identidad y cambio, Bellaterrra, Barcelona.

Esteban, Mari Luz

2011 Crítica al pensamiento amoroso, Bellaterra, Barcelona.

Fagetti, Antonella

1998 Tentzonhuehue, el simbolismo del cuerpo y de la naturaleza, Benemérita Universidad Autónoma de Puebla/Plaza y Valdés, México.

FoucAult, Michel

1988 "L'incorporation de l'hôpital dans la technologie moderne”, en Hermès, núm. 2, pp. 30-40.

Freud, Sigmund

1970 "Frammento di un'analisi d'isteria (Caso clinico di Dora)", en Opere IV, Bollati Boringhieri, Turín.

Freud, Sigmund

1977 Pulsiones y destinos de pulsión, en Obras completas, t. XIV. Amorrortu, Buenos Aires.

Freud, Sigmund

1985 Casi clinici. Vol. 3. Dora, Bollati Boringhieri, Turín.

Freyermuth, Graciela

y María de Jesús Montes

2000 "Parteras tradicionales en el estado de Chiapas, usos y costumbres", en Natura Medicatrix, núm. 57, pp. 20-25.

Gamio, Manuel

1922 La población del valle de Teotihuacán. El medio en que se ha desarrollado, su evolución étnica y social, iniciativas para procurar su mejoramiento, Dirección de Talleres Gráficos, México.

Gaskin, Ina MaY

2002 Spiritual Midwifery, Book Publishing, Summertown.

Gervás, JuAn

y Mercedes PÉREZ

2015 La expropiación de la salud, Sin Fronteras, Barcelona.

Gimbutas, MariJa

1989 The Language of the Goddess, Harper Collins Publisher, Nueva York.

Gonzales, Patrisia

2012 Red Medicine: Traditional Indigenous Rites of Birthing and Healing, The University of Arizona Press, Tucson.

GoOd, BYron J.

2006 Un corpo che soffre. La costruzione di un mondo di dolore cronico, en Ivo Quaranta (ed.), Antropologia medica. I testi fondamentali, Raffaello Cortina, Milán, pp. 235-264.

GOOD, BYRON J.

y Mary-Jo DelVecchio Good

1982 "Toward a Meaning-Centered Analysis of Popular Illness Categories: 'Fright Illness' and 'Heart Distress' in Iran", en Anthony J. Marsella y Geoffrey M. White (eds.), Cultural Conceptions of Mental Health and Therapy, Reidel (Culture, Illness, and Healing 4), Dordrecht, pp. 141-166.
JiMÉNEZ, ROSALINDA

2013 "Elementos histórico-antropológicos acerca de la partería en México", en Miguel Ángel Adame Cerón (comp.), Ecosalud y antropología de las medicinas alternativas y tradicionales, t. I, Ediciones Navarra, México, pp. 143-156.

Juliano, DOLORES

2017 Tomar la palabra: mujeres, discursos y silencios, Bellaterra, Barcelona.

Kleinman, ARthur

1988 The Illness Narratives: Suffering, Healing, and the Human Condition, Basic Books, Nueva York.

Lagueur, Thomas W.

1994 La construcción del sexo. Cuerpo y género desde los griegos hasta Freud, Crítica, Madrid.

LEBOYER, FRÉDÉRICK

1974 Pour une naissance sans violence, Éditions du Seuil, París.

LEÓN, NiCOLÁs

1910 La obstetricia en México. Notas bibliográficas, etnicas, históricas, documentarías y criticas de los orígenes históricos hasta el año 1910, Tipografía de la Vida, México.

LiEDLOFF, JEAN

2009 El concepto de continuum. En busca del bienestar perdido, Ob Stare, Tenerife [2003].

López Austin, Alfredo

1980 Cuerpo humano e ideología. Las concepciones de los antiguos nahuas, Universidad Nacional Autónoma de México-Instituto de Investigaciones Antropológicas, México, 2 vols.

LuCiani, Morena

2008 Donne sciamane, Venexia, Roma.

Luna, Marisol, José Muños y Graciela Freyermuth 2017 Numeralia 2015. Mortalidad materna en México, Observatorio de Mortalidad Materna en México/Centro de Investigaciones y Estudios Superiores en Antropología Social, México.

Martin, EMily

1987 The Women in the Body. A Cultural Analysis of Reproduction, Beacon Press, Boston.

MenÉndez, EDUARDo L.

1980 Clases subalternas y el problema de la medicina denominada "tradicional", Centro de Investigaciones y Estudios Superiores en Antropología Social (Cuadernos de la Casa Chata, 32), México.

Menéndez, EduARdo L.

1991 "Definiciones, indefiniciones y pequeños saberes", en Alteridades, año 1, núm. 1, pp. 21-32.

Moore, Henrietta L.

1994 A Passion for Difference: Essays in Anthropology and Gender, Indiana University Press, Bloomington.

Moreno, JACOBo L.

1977 Teatro de la espontaneidad, Vancu, Buenos Aires.

Murguialday, Clara, Karlos Pérez de Armiño

Y MARLEn EizaguirRe

2001 "Empoderamiento", en Karlos Pérez de Armiño (dir.), Diccionario de Acción Humanitaria y Cooperación al Desarrollo, Icaria/Hegoa, Bilbao <http: / /www.dicc.hegoa.ehu.es /

OAKLEY, ANN listar/mostrar/86> [5 de diciembre de 2017].

1985 "Il parto: un evento biosociale", en Ann Oakley et al., Le culture del parto, Feltrinelli, Milán, pp. 3-12. 
ODENT, MiChel 1984 Birth Reborn, Random House, Nueva York.

Odent, Michel 1992 Il bebè è un mammifero, Red Edizione, Roma.

OLZA, IBONE

Y ENRIQUE LEBRERO

2005 ¿Nacer por cesárea? Evitar cesáreas innecesarias, vivir cesáreas respetuosas, Granica, Guadalajara, México.

Pichón Rivière, EnRIgUe

Y ANA PAMPliEga

2002 Psicología de la vida cotidiana, Nueva Visión, Buenos Aires.

Quagliariello, Chiara

2014 "Modelli culturali della nascita a confronto: L'evento parto tra le donne della comunità senegalese di Poggibonsi”, tesis de doctorado, Università degli Studi di Siena.

QuARANTA, IVo (ED.)

2006 Antropologia medica. I testi fondamentali, Raffaello Cortina, Milán.

Quattrocchi, Patrizia

2011 Corpo, riproduzione e salute tra le donne maya dello Yucatán (Messico), Pacini, Pisa.

Regalia, Anita

\section{y Giovanna Bestetti}

2010 "La via chirurgica alla nascita: il caso Italia", en Irene Maffi (ed.), Annuario di Antropologia, n. 12, Nascita, Ledizioni, Milán, pp. 121-147.
Rodrigáñez Bustos, CAsilda

1995 La represión del deseo materno y la génesis del estado de sumisión inconsciente, Madre Terra, Madrid.

Rodrigáñez Bustos, CASILdA

2007 Pariremos con placer. Apuntes sobre la recuperación del útero espástico y la energía sexual femenina. En el 50 aniversario de la muerte de Wilhelm Reich, Crimentales, Murcia.

SCHEPER-Hughes, NANCY

y MARGARET M. LOCK

1987 "The Mindful Body: a Prolegomenon to Future Work in Medical Anthropology", en Medical Anthropology Quarterly, vol. 1, núm. 1, pp. 6-41.

Spitz, René A.

1965 The First Year of Life. A Psychoanalysis Study of Normal and Deviant Development of Object Relations, International Universities Press, Nueva York.

SuÁrez, Liliana, Emma Martín

y Rosalva Aída Hernández

2008 Feminismos en la antropología: nuevas propuestas críticas, Ankulegi, San Sebastián.

Villa Rojas, Alfonso

1985 Estudios etnológicos. Los mayas, Universidad Nacional Autónoma de México, México.

Weber, Esther

2013 Portare iPiccoli: Un modoantico, moderno...e ecomodo per stare insieme, Il Leone Verde, Turín. 